\title{
Effect of plastic slip on thermomechanical behavior of NiTi polycrystals investigated by micromechanics modelling
}

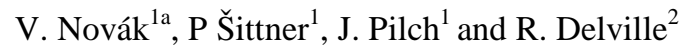 \\ ${ }^{1}$ Institute of Physics ASCR, Na Slovance 2, Prague, Czech Republic \\ ${ }^{2}$ EMAT, University of Antwerps, Belgium
}

\begin{abstract}
Earlier developed micromechanics crystallographic model of SMA polycrystals has been modified by incorporating plastic deformation as additional deformation mechanism. It is assumed that dislocation slip proceeds in austenite and $\mathrm{B} 19^{\prime}$ martensite phases in parallel with the processes derived from B2B19'martensitic transformation in NiTi alloy. The model was used to simulate the responses of NiTi polycrystal in three thermomechanical cycles typically carried out in the SMA research. Based on the simulation results, it is proposed that, due to the dislocation slip occurring simultaneously with transformation related processes, the stresses and strains in the transforming polycrystal are significantly redistributed which in turn modifies the macroscopic thermomechanical responses. In tensile tests at low temperature, the plastic deformation occurs in martensite phase after the stress induced transformation or martensite reorientation, while at medium and high temperatures both deformation mechanisms proceed in parallel. Depending on whether the plastic deformation in tensile tests had taken place in the austenite (typically at high temperatures) or in the martensite (typically at low temperatures) phase, distribution of internal stresses is quite different. Surprisingly, significant plastic deformation activity is predicted for the cooling/heating tests under constant tensile stress. Plastic deformation occurs in this case mainly in the martensite state and redistribution of stress plays significant role. Results of the simulation of the cyclic tensile test at constant temperature explain qualitatively most of the features of the unstable superelastic stress-strain curves observed in cyclic loading experiments. It is proposed that the actual reason for accumulation of unrecovered strain during cyclic loading is in fact the continuously evolving distribution of internal stresses, strains and phase fractions - it causes the plastic deformation to progress even if the macroscopic maximum strain remains constant and peak stress decreases upon cyclic loading.
\end{abstract}

\section{Introduction}

The weakest point of NiTi shape memory alloy is probably its relatively low resistance to plastic deformation by slip or twinning [1]. The alloy must be either work hardened by cold working or precipitation hardened by special heat treatment to show functional properties as superelasticity and/or shape memory [1]. Even after such treatment, however, irreversible plastic deformation commonly takes place alongside with the deformation processes derived from martensitic transformation.

Figure 1 shows TEM evidence on dislocation activity in superelastic NiTi wire heat treated by electric current (125W/16ms) subjected to 10 tensile cycles [2]. While the superelastic response of the $125 \mathrm{~W} / 12 \mathrm{~ms}$ treated wire (Fig. 1a) with nanosized microstructure $(\sim 50 \mathrm{~nm})$ is quite stable [3], unrecovered strain increases up to $3 \%$ upon tensile cycling of the $125 \mathrm{~W} / 16 \mathrm{~ms}$ treated wire with grain size $\sim 400 \mathrm{~nm}$ (Fig. 1b). Figure 1c clearly documents dislocation activity on $\{110\}$ slip systems within one of the larger grains of the $125 \mathrm{~W} / 16 \mathrm{~ms}$ treated wire. Burgers vectors of dislocations were identified to be $\langle 100\rangle$ by the application of the $\mathbf{g . b}=0$ extinction criterion [2]. Figure 1d shows an overview suggesting that the $\langle 100>/\{011\}$ slip system activity is distributed very unequally among the grains. Extinction contours along grain boundaries evidence the presence of internal stresses in mechanically cycled wire. Based on this evidence, we assume that the NiTi wire deforms by $<100>/\{011\}$ slip in addition to stress induced martensitic transformation and that significant internal stresses are introduced in the material after just a few superelastic stress-strain cycles.

Plastic deformation during cyclic deformation of NiTi brings about accumulation of permanent strain upon cycling and drift of functional characteristics (e.g. hysteresis width or transformation strain). This functional fatigue is definitely one of the biggest issues for engineering applications of NiTi. On the other hand, other SMA alloys which are more resistant against plasticity as $\mathrm{NiMnGa}$, CoNiAl or CuAlNi frequently fail by intergranular fracture in polycrystalline state.

a e-mail: novakv@fzu.cz

This is an Open Access article distributed under the terms of the Creative Commons Attribution-Noncommercial License (http://creativecommons.org/licenses/by-nc/3.0/), which permits unrestricted use, distribution, and reproduction in any noncommercial medium, provided the original work is properly cited 
It thus appears that limited amount of dislocation activity is in fact beneficial for stable SMA functional properties. Dislocation slip, however, severally affects the performance of the alloy, particularly the functional fatigue. Controlling the extent of plastic deformation in NiTi is hence essential for engineering applications requiring stable performance and good fatigue resistance. It can be made through careful microstructure control through hot/cold working and heat treatments. The nonconventional heat treatment of cold work hardened NiTi wire by electric current [4] can be used to adjust the functional superelastic properties of the alloy, particularly the transformation strain, hysteresis width, strength and stability in cyclic deformation (Fig. 1) Hence, in order to be able to select best heat treatment conditions, we need to understand the mechanics of NiTi polycrystal undergoing simultaneously the martensitic transformation and plastic deformation. This is best done through mechanics modelling.
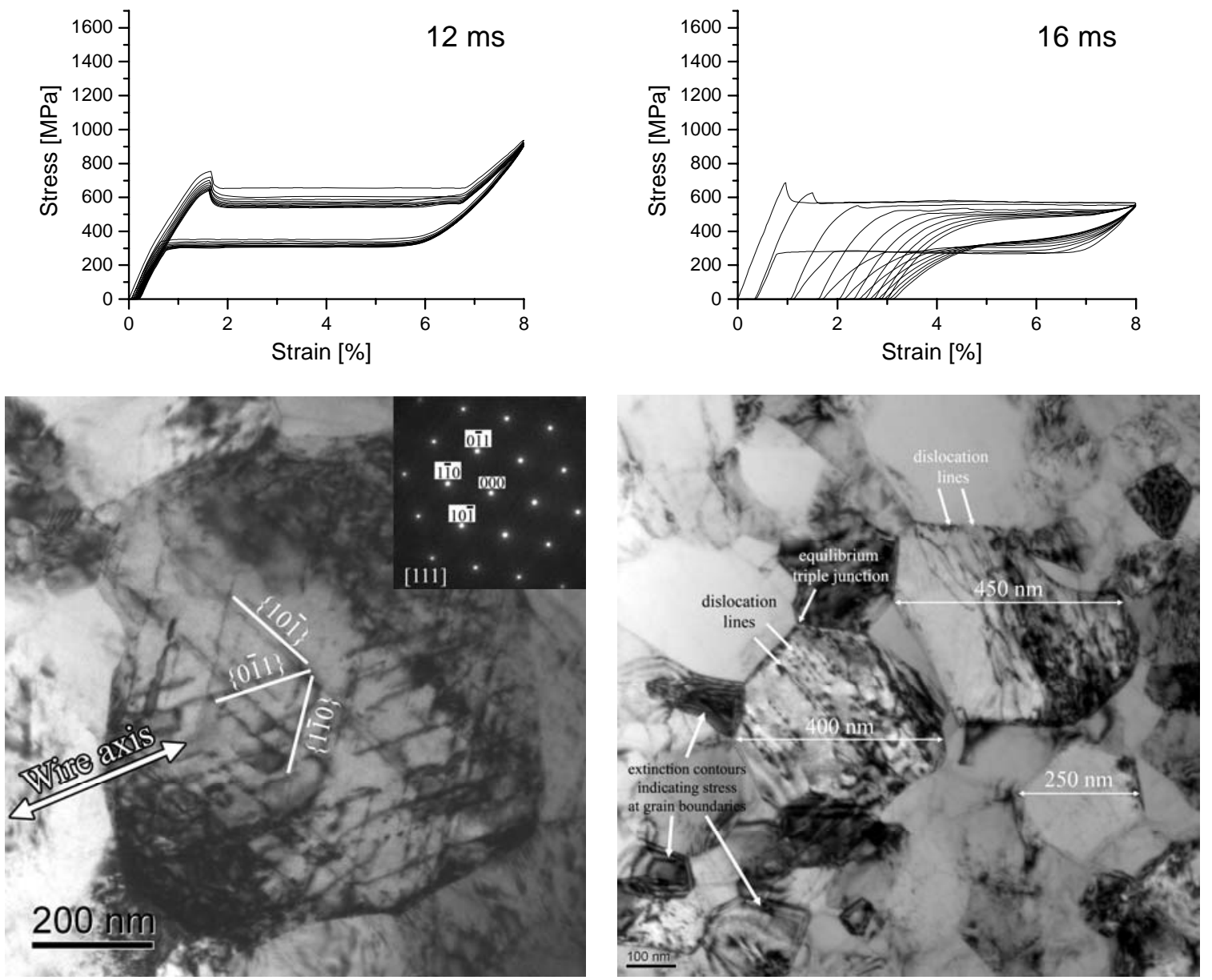

Fig. 1. Evidence for permanent plastic strain $(a, b)$, dislocation activity on $\{110\}$ slip systems $(c, d)$ and internal stresses (d) (extinction contour along grain boundaries due to strain mismatch between grains) in superelastic NiTi wire subjected to 10 tensile cycles (Fort Wayne Metals \#1 0,1mm thin wire heat treated by electric current pulse $125 \mathrm{~W} / 12 \mathrm{~ms}$ (a) and $125 \mathrm{~W} / 16 \mathrm{~ms}(\mathrm{~b}, \mathrm{c}, \mathrm{d})[2,3])$.

Plasticity shall thus be viewed as another deformation mechanism to be considered in SMA modelling. Few researchers attempted that before on continuum mechanics level [5]. The continuum mechanics models can possibly reproduce the unstable experimental stress-strain curves upon cycling, nevertheless, predictions for the best heat treatments can be hardly made based on these models without understanding the coupling between transformation and plasticity in constrained polycrystal environment. Micromechanics models [6,7] which take into account the crystallographic details and anisotropy of dislocation slip as well as martensitic transformation have a better chance for success in this direction. Problem is that there is not enough experimental information available on plastic deformation of NiTi. Basic slip parameters as critical resolved shear stress and hardening coefficients are known only for B2 austenite phase [8] but not for the B19' martensite phase. A coupling between plasticity and transformation in NiTi on crystal lattice level (as developed e.g. for TRIP steels) has been proposed very recently by Norfleet et al [9] who suggested a possibility of cooperative transformation-plasticity in NiTi with involvement of the B2 <100>/\{011\} slip. 
It will be shown in this work that combination irreversible plastic deformation with reversible martensitic transformation in modelling of NiTi polycrystals subjected to thermomechanical loads can qualitatively explain very complex phenomena, even if we neglect any direct coupling between phase transformation and plasticity. An earlier developed micromechanics model of SMA polycrystals [7,10,11] which captures activity multiple deformation mechanisms in phase transforming materials is purposely modified by incorporating plastic deformation as additional deformation mechanism. Three basic thermomechanical loads were simulated: i) tensile tests, ii)cooling/heating under constant stress, iii) cyclic tensile test. For simplicity, the simulations are limited to tensile deformation only, results for compression or other deformation modes are different.

\section{SMA polycrystal model update}

\subsection{Micromechanics crystallographic model of SMA polycrystals}

In the micromechanics crystallographic model, SMA polycrystals has been modeled [7,10,11] as a networked aggregate of interacting oriented domains (Fig. 2a) in which a uniaxial homogeneous stress state exists. Rate independent deformation mechanisms yielding elastic strains, thermal dilatation and strains due to multiple transformation processes are considered to proceed in individual domains depending on the stress-temperature conditions there. Thermomechanical responses of individual domains are modeled using a concept of so called non-equilibrium $\sigma-T$ diagrams (Fig. 2b) calculated for each domain from basic material, thermodynamic and crystallographic parameters [7,10]. The austenite transforms under stress into the martensite lattice correspondent variant providing maximum transformation strain into the load axis direction calculated from lattice parameters. It is through the orientation and sense of the load dependence of these maximum transformation strains, how the anisotropy and asymmetry is introduced into the calculated $\sigma-T$ diagrams. These diagrams provide for each particularly oriented domain a complete information on the stresses and temperatures $[7,10]$ at which individual multiple deformation/transformation processes take place. Constitutive responses of individual domains are calculated in a simplest way assuming constant stress (temperature) during transformation [7,10]. Main advantage of the $\sigma-T$ diagrams concept is that all problems with the complex path and history dependencies accompanying the deformation and transformation processes can be rigorously solved on the domain level.
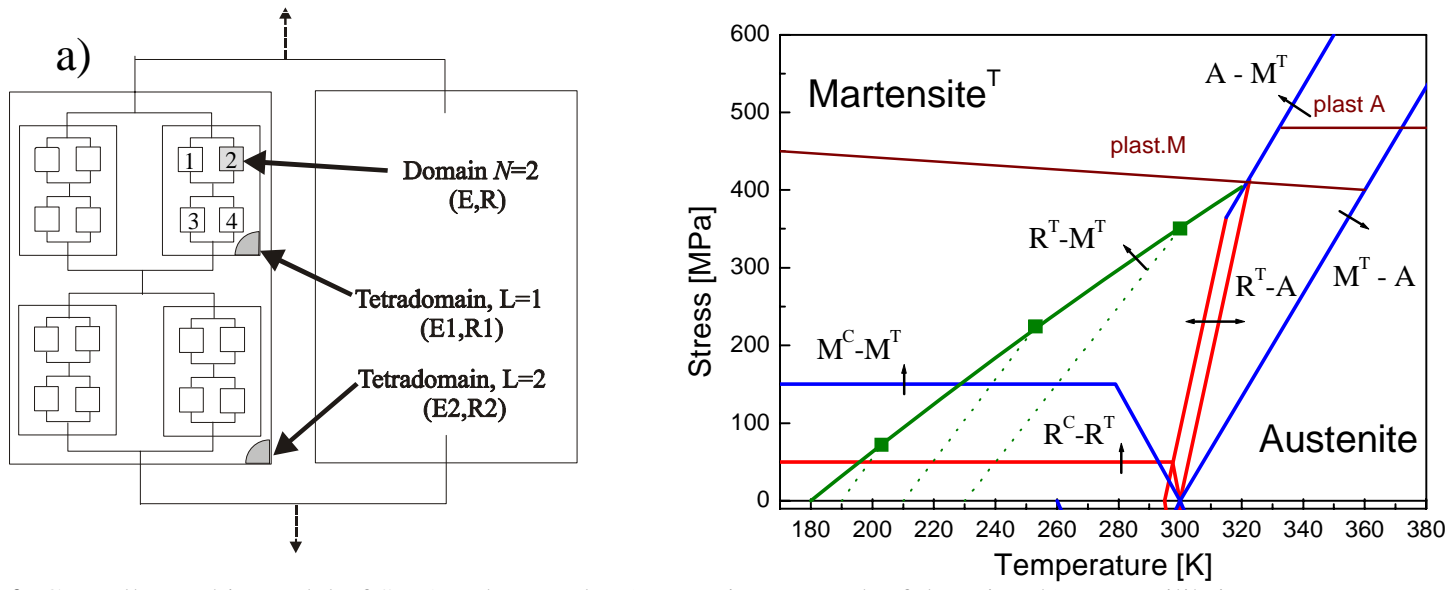

Fig.2. Crystallographic model of SMA polycrystals. a) recursive network of domains, b) nonequilibrium stress-temperature diagram for NiTi transforming via B2_RB19' [11] with extra lines for Yield stress in martensite (plast M) and austenite (plast A) phases.

The polycrystal aggregate is subjected to a thermomechanical load test in which the time evolution of macroscopic stress, $\sigma_{G}$, (or strain, $\varepsilon_{G}$ ) and temperature $T$ is prescribed. The stresses, strains and phase fractions in all domains are calculated as a model response using a recursive network method [10]. There are four substeps in the recursive network calculations, in which the stresses, strains, moduli and phase fractions in all domains are adjusted. Main role of the role of the recursive network is to ensure that the macroscopic stress (strain) imposed on the aggregate is properly redistributed among individual domains. The artificial constraints imposed on uniaxial stresses and strains in this approach are believed to model in reasonable approximation the otherwise very complex multiaxial constraints affecting the progress of martensitic transformation in the SMA polycrystals. The simple strain compatibility conditions and simple scale transition to the polycrystal level is necessary to capture the consequences of the simultaneous activity of many different deformation/transformation processes in the polycrystalline material. 


\subsection{Incorporation of dislocation plasticity into the model}

It is assumed that dislocation plasticity takes place by the slip of dislocations with Burgers vectors $\langle 100\rangle$ on $\{011\}$ crystal planes in austenite phase and with Burgers vectors $\langle 110\rangle$ on $\{111\}$ crystal planes in martenite phase. hkl indexes refer to the cubic austenite phase. Critical shear stress for plastic deformation in a domain $\mathrm{n}$ is calculated by Schmidt law from the known load axis orientation and phase state. Plastic deformation term $\varepsilon_{\mathrm{pl}}$ which is added to the constitutive equation of the SMA polycrystal model (Eq.1) describes plastic deformation either in austenite or in martensite phase, depending on temperature and volume fraction of the martensite $\xi^{\mathrm{n}, \mathrm{p}}$ in that particular domain (Fig. 2b).

$$
e^{n}=\frac{\sigma^{n}}{E^{n}}+\alpha^{n}\left(T-T_{s}\right)+\sum_{p} \varepsilon^{t r, p} \xi^{n, p}+\varepsilon_{p l}
$$

where $e^{n}$ is total deformation of the domain n, $\sigma^{n}$ is stress in the domain, $E^{n}$ is Young's modulus equal for austenite and martensite, $\alpha^{n}$ coefficient of thermal expansion equal for austenite and martensite, $\mathrm{T}$ is temperature, $T_{s}$ the starting temperature in the test, $\mathcal{E}^{t r, p}$ the transformation strain of the process $\mathrm{p}$ and $\xi^{n, p}$ is the volume fraction of martensite phase/variant p. The stress ${ }^{\sigma_{y}}$ for plasticity is assumed to increase linearly with increasing plastic strain due to the deformation hardening (Eq. 2),

$$
\sigma_{y}=\frac{\tau_{C R S S}}{S F}+C_{w h} \varepsilon_{p l}
$$

where $\tau_{C R S S}$ is temperature dependent critical resolved shear stress, $S F$ is the Schmidt factor, and $C_{w h}$ the hardening coefficient. As a result of the hardening, the stress-temperature diagrams changes during the simulation - plasticity lines in figure $2 \mathrm{~b}$ move upwards. Material parameters of NiTi with suppressed R-phase were selected as given in Table 1. Otherwise the model used for simulations below is the same as that described in papers $[7,10,11]$

Table 1 Material parameters of NiTi used in the simulations

\begin{tabular}{|l|l|l|l|l|l|l|}
\hline Test & $\mathbf{M s}$ & $\mathbf{A f}$ & $\mathbf{\Delta S A - M}$ & $\tau^{A}{ }_{\text {CRSS }}$ & $\tau^{M}{ }_{\text {CRSS }}$ & $\mathbf{C}_{\mathbf{w h}}$ \\
\hline Tensile and thermal tests & $293 \mathrm{~K}$ & $313 \mathrm{~K}$ & $0,6 / \mathcal{E}^{\text {tr }}$ & 150 & 130 & 0005 \\
\hline Tensile cyclic test & $243 \mathrm{~K}$ & $263 \mathrm{~K}$ & $0,6 / \varepsilon^{t r}$ & 150 & 130 & 0,005 \\
\hline
\end{tabular}

\section{Simulation results}

Results of the simulations of the NiTi polycrystal response in three basic thermomechanical tests: i) tensile tests (section 3.1), ii) cooling/heating under constant stress (section 3.2) and iii) cyclic tensile test (section 3.3) are shown and discussed below. After each simulation test, simulation of stress free heating above $\mathrm{A}_{\mathrm{f}}$ and cooling back to room temperature is performed to evaluate the stability of the model with respect to such thermal excurse. In real material, this would correspond to the investigation of the stability of internal stresses introduced into material by previous thermomechanical loading. The results are presented in section 3.4.

\subsection{Tensile stress-strain test}

Simulations of four tensile tests at temperatures $\mathrm{T} 1=270 \mathrm{~K}, \mathrm{~T} 2=320 \mathrm{~K}, \mathrm{~T} 3=370 \mathrm{~K}$, and $\mathrm{T} 4=420 \mathrm{~K}$ were made. in order to investigate the effect of starting microstructure. Each simulation test starts by stress free cooling from the room temperature to the test temperature, then tensile loading-unloading is simulated. The loading is reversed when total plastic deformation reaches same value of 5\%. Results of the simulation tests are shown in figure 3. In the $\mathrm{T} 1$ test, the starting microstructure is twinned martensite, in the T2, T3 and T4 tests the starting microstructure is austenite but as the temperature increases, the plastic deformation of austenite prevails over the martensitic transformation. Information about distribution of stress, strain, martensite variant volume fraction and plastic strain in each particularly oriented domain and time step is available in the simulation results. Average values over sets equally oriented domains are calculated and called component stress, component strain etc. 

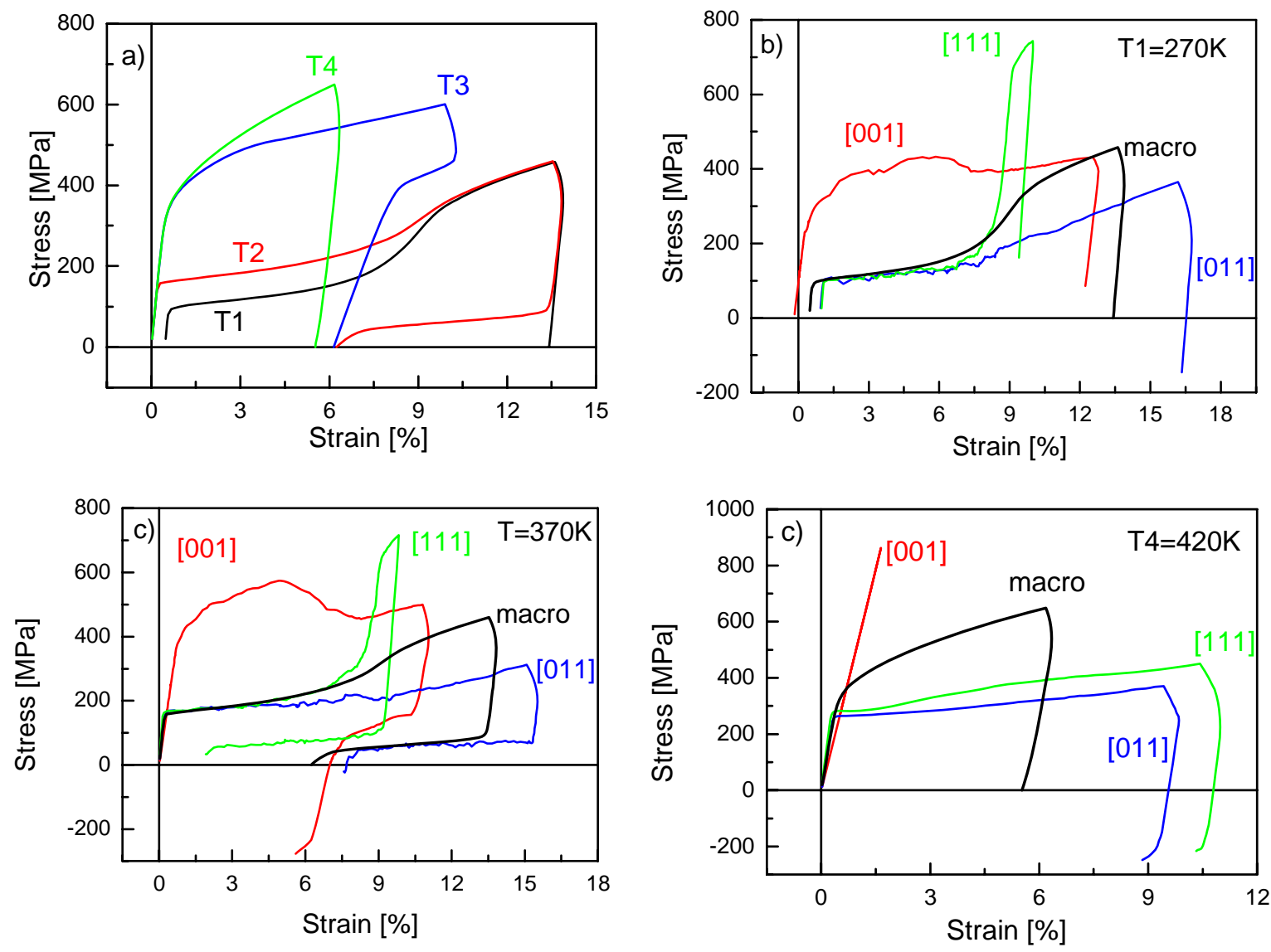

Fig. 3. Simulation results for four tensile tests at constant temperatures $\mathrm{T} 1=270 \mathrm{~K}, \mathrm{~T} 2=320 \mathrm{~K}, \mathrm{~T} 3=370 \mathrm{~K}$, and $\mathrm{T} 4=420 \mathrm{~K}$. a) macroscopic stress-strain curves, b) component stress-strain responses for [001], [011] and [111] families of equally oriented grains in tests T1 (b), T3(c) and T4 (c).

In the $\mathrm{T} 1$ test, the deformation proceeds by reorientation of $\mathrm{B} 19^{\prime}$ ' martensite (compression to tensile variants) followed by plastic deformation in all domains. In case of T2 test, stress induced B2-B19' transformation is followed by plastic deformation of martensite. Significant deformation is recovered upon unloading due to reverse martensitic transformation and ne direct coupling with plastic deformation. In test T3, some domains transform and some deform plastically in austenite, while in test T4 most of the domains deform plastically in austenite. Information on the evolution of stresses and strains in various families of equally oriented domains in is presented in figures $2 \mathrm{~b}-\mathrm{d}$. Note that the distribution of stresses and strains is very different in low and high temperature tests -e.g. the [001] oriented domains deform plastically only in the martensite phase while [111] oriented grains mainly in the austenite phase. After unloading some domains remain under tensile (e.g. [001]) and some (e.g. [011]) under compressive residual stresses. Since the slip geometry is different in the austenite and martensite phases, distribution of internal stress is different in simulation tests at low and high temperatures.

\subsection{Cooling/heating under constant tensile stress}

Thermal cycles were simulated at constant tensile stress 100MPa, 200MPa, 300MPa. Simulated macroscopic strain-temperature tests are shown in figure $4 \mathrm{a}$. While the strains are nearly reversible in cooling/heating cycle under $100 \mathrm{MPa}$, large unrecovered strain is obtained at 300MPa. The component strain-temperature responses at $300 \mathrm{MPa}$ (Fig. 4b) show that the strain is unequally distributed among families of equally oriented domains (grains). Figure $3 \mathrm{c}$ shows the evolution of stress in these families of domains during thermal cycle. In the austenite phase the stress is distributed due to elastic anisotropy. As the martensite forms upon cooling, the stress redistributes significantly among the domains (grains) due to the strong transformation anisotropy (see figure $4 \mathrm{~d}$, 4e). Tensile stress increases particularly on [001] oriented grains and decreases on [011] oriented domains. Since the yield stress for plasticity is lower in the martensite phase and has different anisotropy, many of the domains start to deform plastically and the stress redistributes again. This gives rise to the complex evolution of component stresses presented in figure 4c. With increasing applied stress, the plastic deformation of martensite during cooling significantly increases. As a result, the strain-temperature curves at higher applied stresses are not 
closed. Plastic deformation is hence essentially driven by phase change (lower yield stress) and increase of stress in some domains with decreasing temperature (redistribution of stress). Note that the plastic deformation increases both during cooling and heating (Fig. 4d).
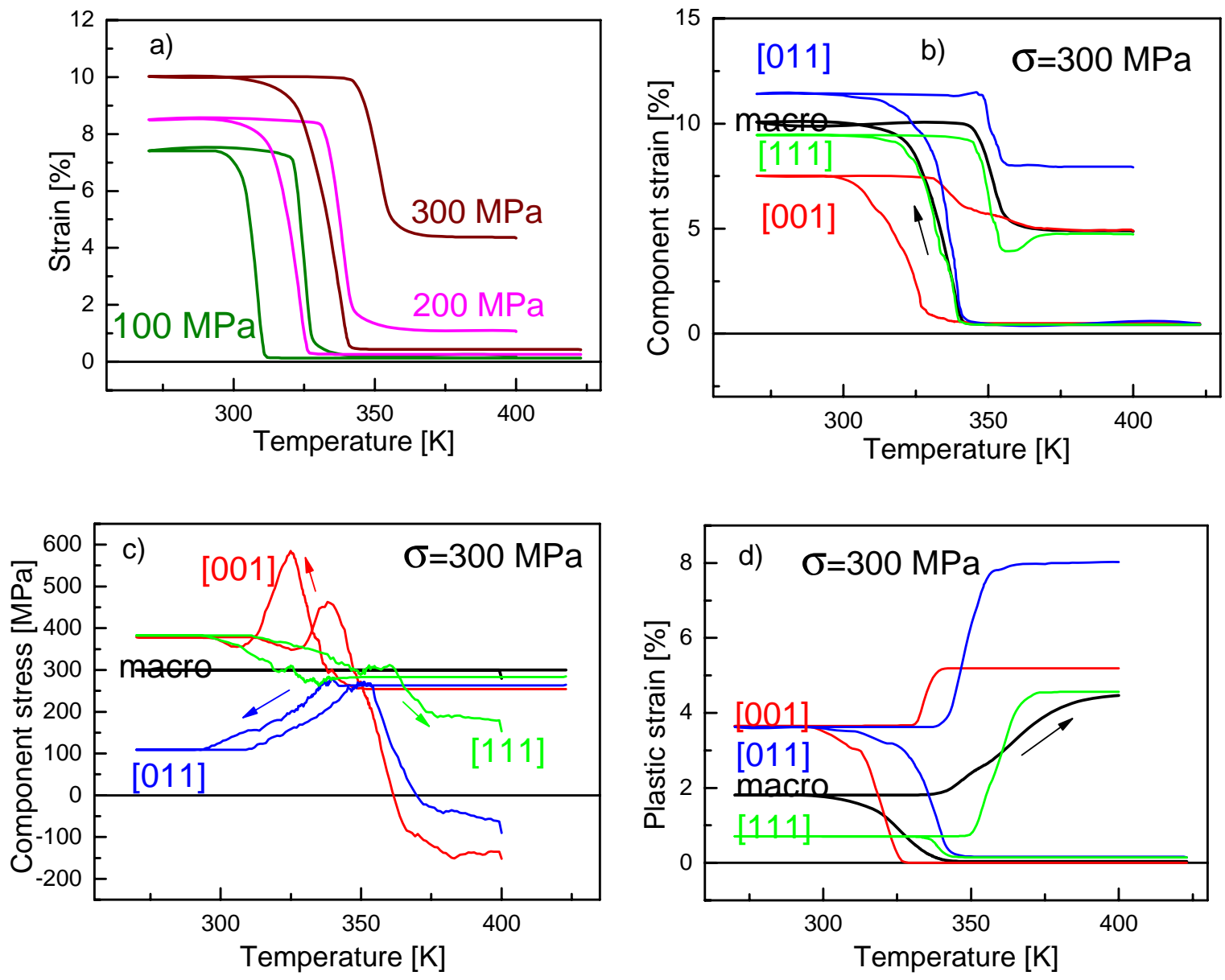

Fig. 4. Simulation results for cooling/heating tests at constant tensile stress $100 \mathrm{MPa}, 200 \mathrm{MPa}, 300 \mathrm{MPa}$, 400MPa. a) macroscopic strain-temperature curves, b) component strain-temperature curves in test at $300 \mathrm{MPa}, \mathrm{c}$ ) component stresstemperature curves in test at $300 \mathrm{MPa}$, d) plastic strain-temperature curves in test at $300 \mathrm{MPa}$ (c)

\subsection{Cyclic tensile test}

Cyclic tensile test (10 cycles) was simulated at temperature $T=A_{f}+27 K$ where superelasticity is expected (Fig. 5a). The loading was reversed at $8 \%$ total strain. The stress induced martensitic transformation was by far the prevailing deformation mechanism in this simulation test but the stress in some domains was so high that plastic deformation marginally took place there. Note that, in spite of so many drastic simplifications adopted in modelling, the simulated stress-strain response is qualitatively very similar to the experimental record in figure 1b. There is no stress plateau in the simulation since there is no way to consider localization of deformation into shear bands with this type of model [14].

It is particularly interesting to see the evolution of component stress (Fig. 5b), plastic strain (Fig. 5c) and martensite volume fraction (Fig. 5d) during cycling. Again the stress is redistributed due to transformation anisotropy in such a manner that maximal tensile stresses exist in [001] domains. However, as the plastic deformation takes place during cycling this redistribution is slowly changing its character due to the plastic deformation events irreversible upon unloading. Note that the plastic strain slightly decreases upon unloading (Fig. 5c). This is due to the compressive deformation occurring in some domains upon unloading (Fig. 5b). It shall be noted that, the plastic strain accumulates upon cycling, even if macroscopic forward stress and component forward stresses decrease during cycling. But then why the plastic strain should accumulate in peak strain is constant and peak stress decreases? It comes out that this is because the macroscopic and component stresses are average values which does not exclude that local stresses in selected individual domains still increase causing further plastic deformation events and accumulation of plastic deformation upon cycling. 

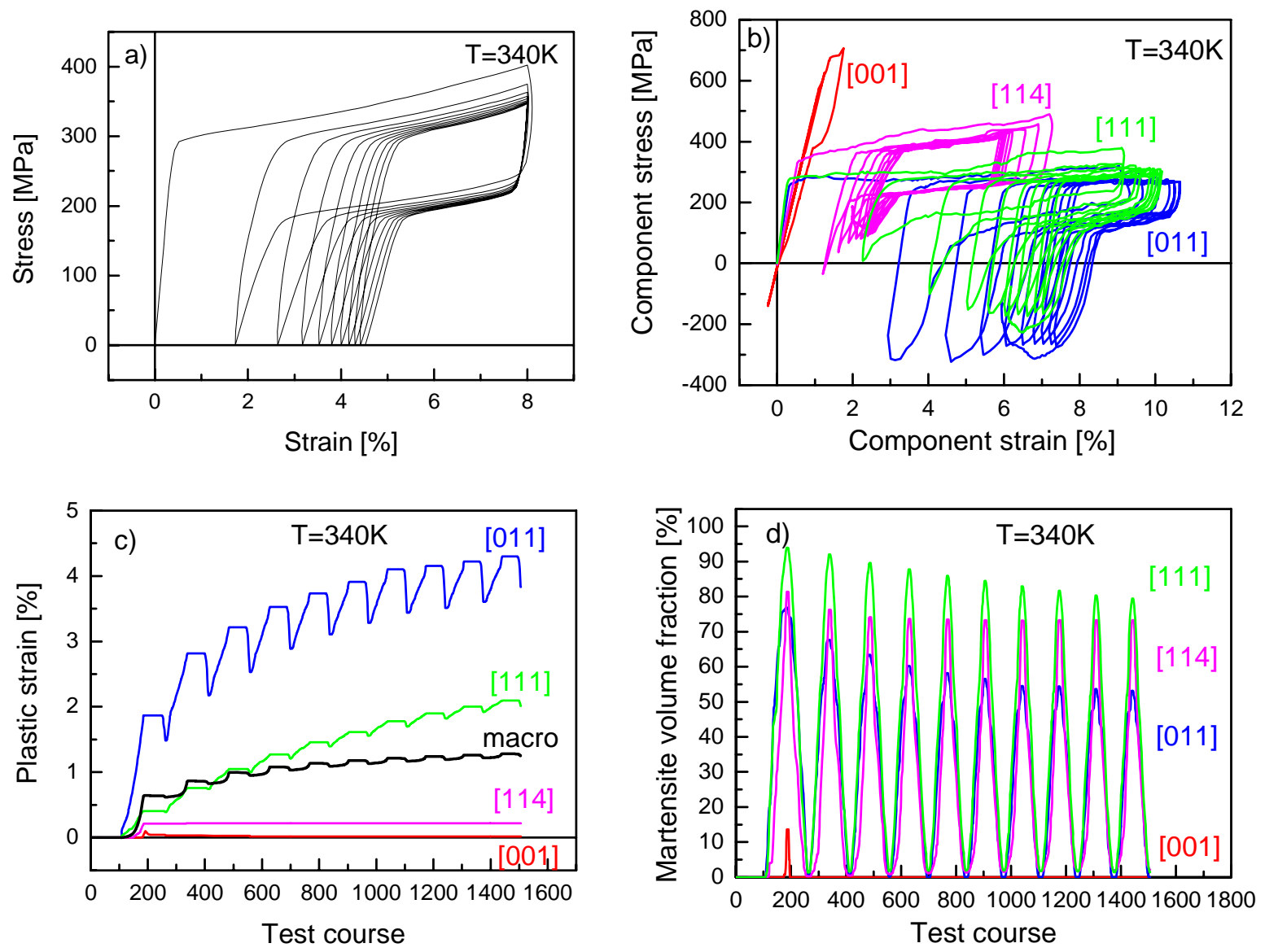

Fig. 5. Simulation results for cyclic tensile test at $T=A f+27 K$, component stress-strain curves (b), component plastic stressstrain curves (c), and component martensite volume fraction evolution during cycling (d),

Plastic strain in various families of oriented domains increases with very different rates during cycling (Fig. 5c). Maximum plastic strains exist in [011] domains, negligible in [001] domains. Volume fraction of martensite varies cyclically (Fig. 5d). The amplitude is very different for differently oriented domains and decreases during cycling. In [001] domains, martensite volume fraction is very small and quickly decreases to zero. Since the plastic deformation in [001] domains at low stress can occur only in martensite phase, the plastic deformation there is negligible.

Volume fraction of martensite at zero macroscopic stress (stabilized martensite) also accumulates during cycling up to $11 \%$ after $10^{\text {th }}$ cycle. The permanent strain left behind after tensile cycling (Fig. 5a) is thus the sum of plastic strain (Fig.5c) and transformation strain of the stabilized martensite. The stabilization of martensite is due to redistribution of stress caused by plastic deformation which leaves some domains under residual local stress at zero macroscopic stress. The condition for reverse transformation $\left(\mathrm{M}^{\mathrm{T}}-\mathrm{A}\right.$ in figure $\left.2 \mathrm{~b}\right)$ is not fulfilled locally in these domains, though it is fulfilled macroscopically. This residual stress keeps the martensite phase present in those domains at temperature above $\mathrm{A}_{\mathrm{f}}$ even at zero macroscopic stress. The response of the sample in such material state upon further heating is discussed in the next section.

\subsection{Post deformation effects}

After cyclic deformation and unloading (section 3.3), the sample exhibits unrecovered deformation of about $4.5 \%$, internal stress and nonzero volume fraction of martensite in some domains. Unrecovered strain (plastic and transformation strain) is nearly zero for [001] domains and maximal for [011] and [111] domains. When the aggregate in such material state in is heated in stress free conditions, individual domains are in fact partially constrained by the neighboring domains (Fig. 2a) and behave as SMA element in actuator test - i.e. with increasing temperature, the transformation strain slowly decreases (Fig. 6a), stress increases and martensite fraction decreases (Fig. 6b). In order to decrease the very small martensite volume fraction down to zero, however, overheating at least $50 \mathrm{~K}$ was needed. Upon cooling back, the strain remains nearly constant but 
martensite volume fraction (in some families of domains) increases even at temperatures much above the Ms. The original material state is thus partially restored after this thermal excurse. The reason for only partial restoration is the plastic deformation which took place at high temperature driven by thermally induced internal stress. The plastic deformation events in turn caused further redistribution of internal stress and phase fractions in the aggregate. It thus comes out that neither the plastic strains nor the transformation strains of the deformed aggregate can be fully restored by overheating.
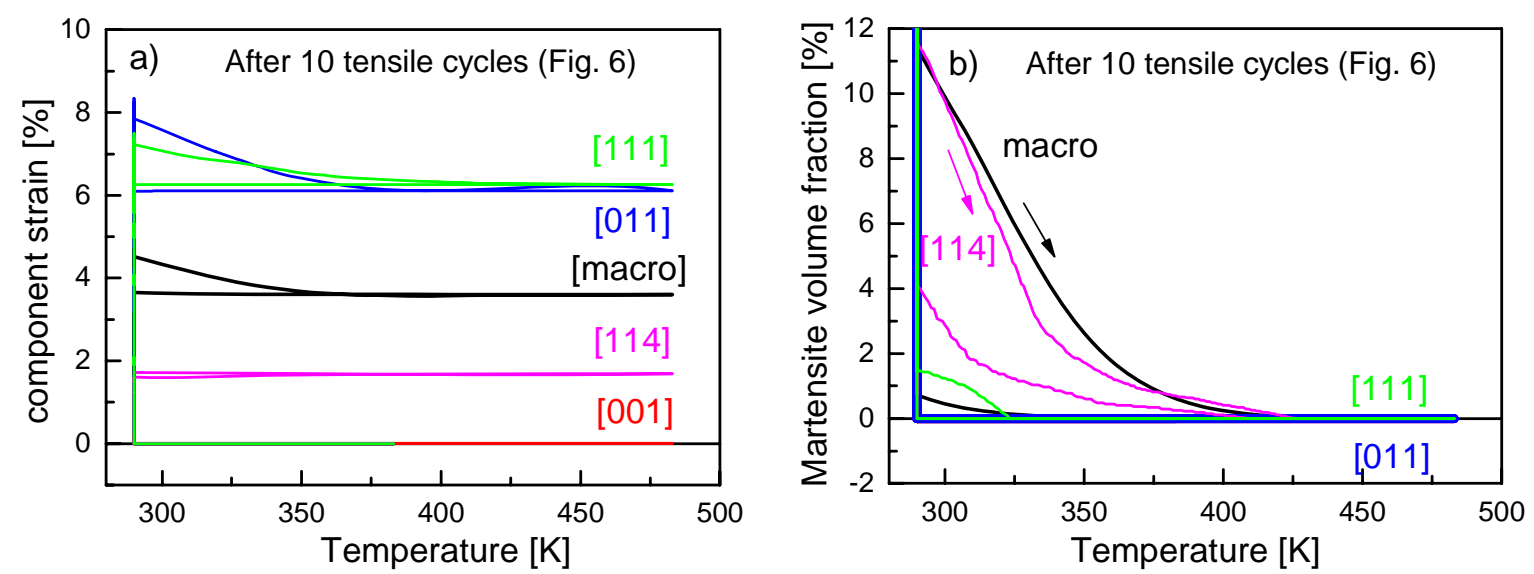

Fig. 6. Simulation results for post deformation heating/cooling after cyclic tensile test at $T=A_{f}+27 K$, a) component straintemperature curves, b) component martensite volume fraction-temperature curves.

Dedicated simulations were made earlier with this model with the aim to explain various phenomena [11,14] or interpret experimental data [12,13] related to martensitic transformations in SMAs. In this work, the model is extended to capture plastic deformation as additional deformation mechanism and basic SMA thermomechanical responses are focused. Main lesson we learned practically from all the presented simulations is that if the plastic deformation takes place alongside with the phase transformation and/or martensite reorientation, the stresses, strains and phase fractions redistribute among the aggregate domains (polycrystal grains) differently than if only deformation mechanisms derived from martensitic transformation are considered. This has severe consequences on the mechanics of the deformation of the SMA aggregate (polycrystal), in particular: i) generation of large internal stresses in the material after unloading, ii) significant plastic deformation during cooling/heating under constant stress, iii) accumulation of unrecovered strain during cyclic tensile tests. Note that these conclusions come out from the modelling even if no coupling between the slip and transformation on crystal lattice level, as suggested e.g. in [9], is considered - i.e. in an unconstrained domain, both deformation mechanisms would proceed in parallel without influencing one another. All phenomena discussed above thus stem solely from the effect of grain boundary constraints on the deformation by simultaneous plasticity and transformation.

\section{Conclusions}

Micromechanics model of SMA polycrystals modified by incorporating plastic deformation as additional deformation mechanism was used to simulate the responses of NiTi polycrystal in three thermomechanical tests commonly used in SMA research (tensile tests, thermal loads under constant stress, cyclic tensile tests). Based on the simulation results, it is suggested that distribution of stresses and strains among grains of the transforming SMA polycrystal due to the anisotropies of elastic, transformation and plastic deformation mechanisms plays a key role in the mechanics SMA polycrystals. It is found that this distribution is very different if dislocation slip occurs alongside with phase transformation processes.

In simulations of tensile tests at low temperatures, the plastic deformation occurs in the martensite phase after the stress induced transformation or martensite reorientation, while at medium and high temperatures both deformation mechanisms proceed in parallel. Depending on whether the plastic deformation in tensile tests had taken place in the austenite phase (typically at high temperatures) or in the martensite phase (typically at low temperatures), distribution of internal stresses is quite different.

Surprisingly, significant plastic deformation activity is predicted for the cooling/heating tests under constant tensile stress. Plastic deformation occurs mainly in the martensite state and it is strongly affected by the redistribution of stress.

Simulation results for cyclic tensile tests at constant temperature explain qualitatively most of the features of the unstable superelastic stress-strain curves observed in experiments. It is proposed that the actual reason for 
accumulation of unrecovered strain during cyclic mechanical loading is in fact the continuously evolving distribution of internal stresses, strains and phase fractions - it causes the plastic deformation to progress even if the macroscopic maximum strain remains constant and macroscopic peak stress decreases upon cyclic loading.

\section{References}

[1] K. Otsuka and X. Ren, Pr. Mat. Sci., 50, 511 (2005)

[2] R. Delville, B. Malard, J. Pilch, P. Sittner, D. Schryvers, in preparation

[3] B. Malard, J. Pilch, P. Sittner, V. Gartnerova R. Delville, D. Schryvers C. Curfs, Functional Materials Letters, 2, 1 (2009)

[4] J. Pilch, L.Heller and P.Sittner, in this volume

[5] P.B.Entchev and D.C.Lagoudas, Modelling of Transformation induced plasticity in SMAs in Shape memory alloys: Modelling and Engineering Applications, p. 233

[6] F. Jemal, T.Bouraoui a,b, T. Ben Zineb, E Patoor, C. Bradad, mechanics of Materials, 41, 849 (2009).

[7] P. Sittner, V. Novak, Int. J. Plasticity 16, 1243 (2000).

[8] H. Sehitoglu, J. Jun, X. Zhang, I. Karaman, Y. Chumlyakov, H. J. Maier and K. Gall Acta mater. 49, 3609 (2001)

[9] D.M. Norfleet, P.M. Sarosi, S. Manchiraju, M.F.-X. Wagner, M.D. Uchic, P.M. Anderson, M.J. Mills, Acta Mater., 57, 3549 (2009).

[10] V. Novak, P. Sittner, Mater. Sci. Eng. A 378, 490 (2004).

[11] P. Sittner, V. Novak, P. Lukas, M. Landa, J. Mech. Eng. Tech. ASME 128, 268 (2006).

[12] V. Novak P. Sittner, G.N. Dayananda, F.M. Braz-Fernandes, K.K. Mahesh, Mat. Sci. and Eng. A 481-482, 127 (2008)

[13] P. Šittner, P. Lukáš, V. Novák, M.R. Daymond, G.M. Swallow, Mat. Sci. and Eng. A 378, 97 (2004)

[14] P. Sittner, Y. Liu, V. Novak, J. Mech. and Phys. of Solids, 53, 1719 (2005) 